\title{
Volatility TRANSMission IN ENERGY Futures MARKETS
}

\author{
Michael Souček ${ }^{a}$, Neda Todorova ${ }^{b}$
}

\author{
${ }^{a}$ Chair of Finance and Capital Market Theory \\ European University Viadrina \\ Große Scharrnstr. 59, 15230 Frankfurt (Oder), Germany \\ Email: soucek@europa-uni.de \\ ${ }^{b}$ Corresponding Author \\ Lecturer, Department of Accounting, Finance and Economics \\ Griffith Business School, Griffith University \\ 170 Kessels Road, Nathan QLD 4111, Australia \\ Phone: +61 73735 7219, Email: n.todorova@griffith.edu.au
}

This study is novel in the application of a multivariate HAR model for studying volatility transmission patterns in energy futures markets. In particular, the nature of volatility spillovers between futures on crude oil, natural gas and gasoil is examined using range-based volatility proxies and splitting volatility in components defined over different time horizons. The results provide evidence that crude oil futures carry significant information for the volatility evolution of other energy futures traded on the ICE and reveal interesting insights in the sources of the documented volatility interrelations. Short-term shocks in Brent oil volatility significantly affect the volatility of gasoil futures, while the impact of oil and gasoil on natural gas is driven by the long-term volatility component. Additionally, Brent oil and gasoil ICE futures volatilities exhibit strong positive dynamic correlation, whereas the remaining pairwise correlation curves are fluctuating around zero.

Keywords: Energy futures; HAR model; volatility transmission; conditional correlations; range-based volatility. 


\section{Introduction}

The last decade was characterized by a continuously enhancing consumption of crude oil and gas, followed by increasing interest in energy related hedging products. Motivated by the recent financial market turmoil, this study analyzes the mechanism of volatility transmission in energy futures markets. Understanding the dynamics of energy commodities and their volatility has become essential since they are broadly used for international diversification investment strategies and in the same time, as mentioned by Vacha and Barunik (2012), tend to exhibit different properties than prices of common financial assets. This is for example due to the fact that prices of energy commodities are influenced by a number of untypical investor types, such as industrial entities and power stations.

This study aims to uncover volatility transmission patterns of actively traded energy futures. Crude oil is an important component of production costs for gasoil, which may suggest the existence of significant co-movements in prices and volatilities of related financial products. Natural gas and crude oil, on the other hand, are close substitutes as an energy source; hence, it is reasonable to assume interdependences between their price fluctuations. Furthermore, volatility transmission between natural gas and oil markets has been established by Ewing, Malik and Ozfidan (2002) based on daily closing values from 1996 to 1999 of indexes that represent the behavior of the stock prices of major companies in the oil and natural gas markets. On the other hand, one may expect that the crude oil futures market, given its size and significance, leads other energy markets. This notion along with the complementary and substitute relationships among energy markers indicates how important it is to analyze the markets for these commodities simultaneously to establish volatility interrelation mechanisms. Understanding the patterns of potential volatility transmission is of practical importance to financial market participants and may be useful for portfolio allocation decisions.

Several studies examine price co-movements of energy commodities and the nature of volatility in these markets. Lanza et al. (2006) study the dynamic conditional correlations in daily returns of WTI oil forward and futures prices based on daily 
price observations between 1985 to January 2004 and find significant correlations and an asymmetric effect in which negative price shocks have a greater impact on volatility. This effect is generally assumed to be related to demand-side shocks. Lin and Tamvakis (2001) focus on the informational linkage of price discovery of crude oil futures contracts on the NYMEX and IPE between 1994 and 1997. Variance spillovers are found to be bi-directional, especially when both markets are trading simultaneously. Based on monthly observations from 1975 to 2009, Ghoshray (2011) studies the persistence of oil price shocks, causal relations between oil prices and macro economic indicators as well as relations between oil prices and agricultural commodities. Using daily and weekly futures data form 1984 to 2001, Pindyck (2004) sheds light on the role of volatility in the crude oil, heating oil, and gasoline markets as well as the determinants of volatility itself showing that changes in price volatility are not predicted by market variables such as inventories or convenience yields whereas changes in volatility affect market variables through the marginal value of storage. Vacha and Barunik (2012) examine market co-movements among crude oil, gasoline, heating oil, and natural gas using a wavelet coherence analysis. Based on daily futures price observations from November 1993 to July 2010, they conclude that heating oil, gasoline and crude oil strongly co-move, whereas natural gas seems to be unrelated to all three other commodities.

In the area of volatility transmission research, Soriano and Climent (2006) summarize main methodologies and results of extant studies. It becomes obvious that along with stylized facts about volatility like persistence and clustering, it is also often observed that volatility is transmitted across markets. However, research on volatility interrelations in energy markets appears to be far less frequent compared to equity and equity index markets. Moreover, literature on volatility spillovers in energy markets utilizes mostly GARCH-based methodology for returns sampled at daily to monthly frequencies. For example, using BEKK, CCC, and DCC-MGARCH models on daily data from 2005 to 2008, Chevallier (2012) finds a significant volatility spillover effect between NYMEX crude oil futures and Zeebrugge natural gas futures as well as dynamic correlations bounded between - 0.3 and 
0.3. Singh et al. (2011) identify various volatility transmission effects between crude oil, heating oil and natural gas in the U.S. futures energy markets using MGARCHBEKK representation for daily returns during the period 1994 to the beginning of 2011. The authors find a bi-directional volatility spillover effect between heating oil and natural gas markets which might be explained by the fact these products are substitutes. Jin et al. (2012) employ daily data from July 2005 to February 2011 for WTI, Dubai and Brent futures contracts with a VAR-BEKK model and show that Brent and Dubai crude are much more responsive to market shocks than WTI.

Further literature studies volatility transmission patterns between energy markets and food (Serra, 2011), US equity and equity markets of Saudi Arabia, Kuwait, and Bahrain (Malik and Hammoudeh, 2007), gold (Ewing and Malik, 2013), currency, equity and other commodity markets (Khalifa et al., 2012) or among major benchmarks in the international oil market (Chang et al., 2010). These studies also employ exclusively GARCH specifications to shed light on the dynamics of volatility transmission patterns.

The main contribution of the current paper to existing literature is a detailed study of volatility interrelations in energy markets by directly modeling volatility dynamics. In particular, the analysis is conducted under recourse to range-based volatility proxies. Range-based volatility estimators are known to be much more efficient that their return-based counterparts sampled at the same frequency. By using non-parametric estimators of ex-post volatility, our analysis differs from extant literature on volatility spillovers since we study volatility co-movements with a flexible multivariate time-series specification for the volatility time series as proposed by Bubák et al. (2011). In particular, this model is a multivariate extension of the heterogeneous autoregressive (HAR) model of Corsi, Mittnik, Pigorsch and Pigorsch (2008) which includes an explicit consideration of the time-varying volatility of volatility by means of a GARCH model. Within this model, the nature of volatility spillovers is examined by conducting pairwise Granger causality tests. Dividing the underlying volatility in components defined over different time horizons leads to valuable insights in the source of volatility co-movements because, compared to 
commonly used MGARCH specifications, a vector HAR (VHAR-)based estimation enables describing short-, middle- and long-term spillover effects separately.

The volatility transmission mechanism is examined among the nearest futures on Brent crude oil, gasoil and natural gas from May 2005 to June 2012 traded on the IPE in London which, acquired by the IntercontinentalExchange (ICE) in 2001, is one of the leading energy futures and options exchanges. Since 2005, when the entire portfolio of energy futures at the ICE became electronic, the daily trading volume of, for instance, Brent crude oil futures increased almost sixfold by 2012 and the open interest grew by $300 \%$.

The main findings of this study can be summarized as follows. The estimation of the vector HAR allows for analyzing the impact of different volatility components, which is novel in the literature on energy markets. The model estimates confirms the interpretation of the Granger causality tests and verify the existence of volatility spillovers on the ICE energy market. The largest impact on energy futures volatility in terms of magnitude arises from the own long-term component for Brent oil and gasoil and from the own mid-term component for natural gas. Brent oil volatility seems to lead the volatility of gasoil and natural gas, which is a reasonable result, since crude oil is a large component of gasoil production costs and natural gas is known to be a potential oil substitute as an energy source. Moreover, gasoil volatility appears to contain additional information for the future natural gas volatility development. The source of causality between Brent crude oil and gasoil is the short-term volatility of Brent. While the middle- and long-term components in gasoil volatility are incorporated in its own time series, short-term shocks are determined solely by fluctuation in crude oil futures prices. This volatility spillover may be interpreted as a reaction of the investors to sudden shocks in production cost. The results indicate that the major source of volatility transmission from Brent oil to natural gas is the long-term volatility component; i.e., acting of long-term agents and the expected future size of trends and risk in the commodity time series, as suggested by Corsi (2009). Similarly, also the causality between gasoil and natural gas volatility can be explained by the medium- and long-term volatility component of gasoil. On the 
other hand, forecasts of Brent oil volatility seem broadly unaffected by the volatility components of its potential substitute. Looking closely at the results, while the interpretation considering natural gas as a substitute of oil is rather valid for the US energy market (Singh, Karali, and Ramirez, 2011, Mu, 2007), the source of the spillovers might be of a different nature in the context of ICE contracts. Brent crude oil is an internationally traded crude oil with its physical base BFOE (Brent Blend, Forties Blend, Oseberg and Ekofisk) being extensively used in the EU. Gasoil, on the other hand, has a physical base which located in the ARA (Antwerp, Rotterdam, Amsterdam) and natural gas has a physical base at the UK NBP (National Balancing Point), where the National Grid clears physical trades for gas coming from a variety of sources including the North Sea (e.g. UK, Norwegian or Dutch production). Natural gas arriving via Interconnector could carry European natural gas from various provenances, including potentially Russian gas priced at the German border using index linking to Brent prices. It follows, that although natural gas is priced on ICE, gas contracts are effectively index-priced on Brent crude oil and gasoil, which could result in significant price and volatility linkages. Additionally, quite a lot of the substitution characteristics between gas and crude (heating oil) in the US market takes place in residential and commercial consumption for heating during the winter. However, the substitution between these two energy sources in Europe is only in electricity generation, where the level substitution is rather low. The gasoil generation tends to be the most expensive and is used last during peaks, rather than to substitute gas.

The analysis of pairwise dynamic conditional correlations is conform to the reported regression results. For all pairs, a time-varying and volatile pattern is present in the correlation structure. The volatilities of natural gas and gasoil futures and natural gas and crude oil do not exhibit a pronounced and sustainable instantaneous correlation despite of these assets being substitutes or linked through market specifics. On the other hand, gasoil which is a main refined of crude oil is shown to have a continuous, very strong positive correlation, as expected.

The article is arranged as follows. The next section presents the methodology 
of the study. The data are described in section 3. In section 4, the empirical results are reported and discussed. Section 5 concludes the paper.

\section{Methodology}

\subsection{Transmission model and time-varying correlation}

To analyze interrelationships among volatility time series, we use a multivariate version of the heterogeneous autoregressive model of Corsi et al. (2008) proposed by Bubák et al. (2011). Motivated by the heterogeneous market hypothesis presented by Müller et al. (1997) which assumes the presence of heterogeneity across traders, Corsi (2009) proposes a simple autoregressive-type model for realized volatility, the HAR model, considering volatilities realized over different periods of time. This specification is based upon the idea that traders with different time horizons cause different types of volatility components. The notion of an asymmetric volatility propagation is supported by the observation that volatility over longer time intervals has a stronger influence on volatility over shorter time intervals than vice versa. In the default univariate version of the model, volatility forecasts are linear functions of the current daily, weekly, and monthly realized volatilities. The parsimonious HAR model is shown to capture successfully the persistence of realized volatility for various forecasting horizons and is used in numerous studies on financial market volatility (Andersen et al., 2007; Ait-Sahalia and Mancini, 2008; McAleer and Medeiros, 2008, among others).

To analyze volatility transmission patterns in energy markets, a multivariate version of the HAR model (VHAR) assuming DCC-GARCH process by Engle (2002) for the residuals is used. The VHAR specification is given by

$$
v_{t}=\beta_{0}+\beta_{1} v_{t-1}+\beta_{5} v_{t-1, t-5}+\beta_{22} v_{t-1, t-22}+\epsilon_{t}
$$

where $\boldsymbol{v}$. is logarithmic volatility vectors. While $\boldsymbol{v}_{\boldsymbol{t}}$ and $\boldsymbol{v}_{\boldsymbol{t}-\mathbf{1}}$ contain volatilties of one day ( $t$ and $t-1$, respectively), the elements of $\boldsymbol{v}_{\boldsymbol{t}-\mathbf{1 , t - 5}}$ and $\boldsymbol{v}_{\boldsymbol{t}-\mathbf{1 , t - 2 2}}$ are the 
standardized volatility sums over the last 5 and 22 days.* Logarithmic volatility is used rather that volatility itself because the distribution of logarithmic volatility is much closer to Gaussian, which is of benefit for statistical purposes.

To investigate the time varying correlation, the DCC-GARCH model of Engle (2002) is employed. The DCC correlation model is based on the decomposition of the conditional covariance matrix into conditional standard deviation and time varying conditional correlation. For the vector of innovation term $\boldsymbol{\epsilon}_{\boldsymbol{t}}$ from the VHAR model, a basic multivariate GARCH model is used, with

$$
\boldsymbol{\epsilon}_{\boldsymbol{t}}=D_{t} \eta_{t} \quad \text { and } \quad Q_{t}=D_{t} \Gamma_{t} D_{t}
$$

where $\eta_{t}$ is a $n \times 1$ vector with $E\left(\eta_{t}\right)=0$ and $E\left(\eta_{t} \eta_{t}^{\prime}\right)=I_{n} . D_{t}=\operatorname{diag}\left(h_{1}^{1 / 2}, \ldots, h_{n}^{1 / 2}\right)$ is a diagonal matrix of conditional standard deviations. The conditional variance $h_{i t}$ is defined as a univariate $\operatorname{GARCH}(1,1)$ process,

$$
h_{i t}=\omega_{i}+\alpha_{i 1} \varepsilon_{i, t-1}^{2}+\beta_{i 1} h_{i, t-1} .
$$

If the $\eta_{t}$ is an i.i.d. vector of random variables, with zero mean and unit variance, $\Gamma_{t}$ is the conditional correlation matrix of the standardized residuals, $\eta_{i t}=\varepsilon_{i t} / \sqrt{h_{i t}}$. The dynamic conditional correlation can be estimated as

$$
\Gamma_{t}=\left\{\operatorname{diag}\left(Q_{t}\right)^{-1 / 2}\right\} Q_{t}\left\{\operatorname{diag}\left(Q_{t}\right)^{-1 / 2}\right\}
$$

where $Q_{t}$ is a $k \times k$ symmetric positive definite matrix given by

$$
Q_{t}=\left(1-d_{1}-d_{2}\right) \bar{Q}+d_{1} \eta_{t-1} \eta_{t-1}^{\prime}+d_{2} Q_{t-1}
$$

$\bar{Q}$ stands for unconditional variance matrix of the $\eta_{t}$. The parameters $d_{1}$ and $d_{2}$ are scalars which capture the effect of previous shocks and previous dynamic correlation on the current conditional correlation. They have non-negative values and should

\footnotetext{
*The volatility proxies used in this study and their estimation are described in the following section.
} 
satisfy $d_{1}+d_{2}<1$. DCC is not linear but can be estimated using a two-step method based on the classical maximum likelihood function. A first step is to estimate the univariate GARCH from equation (3) and in a second step, the correlation coefficients are estimated. For technical details about the estimation, the reader is referred to Silvennoinen and Teräsvirta (2009).

\subsection{Volatility proxies}

The HAR model was originally designed for modeling realized volatility which is based on the root of the sum of squared intraday returns (Andersen and Bollerslev, 1998). Limited by data unavailability at the high-frequency level, this study employs daily data. The unconditional volatility in this case is usually estimated in literature using daily closing prices. However, this estimator is obviously very noisy since it does not consider the information given by the price movements within the period of reference. Extreme value estimators are capable of improving estimation precision to a substantial extent. Range-based estimators are based upon the idea that a price range over a time interval may capture volatility better than the corresponding squared returns because the range arises from the whole observed price process whereas the return data is sampled at fixed time points. Extended research on the evaluation of range-based volatility estimators proves the efficiency of these estimators for various assets and time periods (Alizadeh et al., 2002, Vipul and Jacob, 2007, Jacob and Vipul, 2008, among others).

In this study, the estimators of Parkinson (1980), Garman and Klass (1980) and Rogers and Satchell (1991) are adopted. Assuming a driftless price process, Parkinson (1980) estimates volatility by considering the logs of the daily high $\left(H_{t}\right)$ and low $\left(L_{t}\right)$ prices,

$$
\sigma_{P K, t}^{2}=0.3607\left(H_{t}-L_{t}\right)^{2}
$$

Garman and Klass (1980) extend the estimator of Parkinson (1980) by incorporating 
the $\log$ opening $\left(O_{t}\right)$ and closing prices $\left(C_{t}\right)$,

$$
\begin{aligned}
\sigma_{G K, t}^{2}= & 0.511\left(H_{t}-L_{t}\right)^{2}-0.019\left(\left(C_{t}-O_{t}\right)\left(H_{t}+L_{t}-2 O_{t}\right)\right. \\
& \left.-2\left(H_{t}-O_{t}\right)\left(L_{t}-O_{t}\right)\right)-0.383\left(C_{t}-O_{t}\right)^{2} .
\end{aligned}
$$

Rogers and Satchell (1991) develop an estimator that does not require a zero drift,

$$
\sigma_{R S, t}^{2}=\left(H_{t}-C_{t}\right)\left(H_{t}-O_{t}\right)+\left(L_{t}-C_{t}\right)\left(L_{t}-O_{t}\right)
$$

Furthermore, the estimators presented above are one-period measures. To obtain multi-period volatility measures, necessary for estimating equation (1), we calculate a simple mean over $N$ trading days,

$$
\ln \left(\sigma_{t-1, t-N}\right)=\frac{1}{N} \sum_{i=1}^{N} \ln \left(\sigma_{t-i}\right), \quad N=5,22 .
$$

\section{Data and preliminary analysis}

The current study is based on futures data of Brent crude oil, natural gas and gasoil traded at the ICE. The data cover a period of 7 years and two months between May 2005 and June 2012. Our sample period starts from May 2005 because in April 2005, the entire ICE portfolio of energy futures became fully electronic. Since nearby contracts are traded more actively, and there are contracts maturing every month of the year, they are rolled over at the expirations to obtain a long time-series data. The data are obtained from Datastream and include daily settlement prices, open, high and low prices, open interest and trading volume entries.

To avoid staleness of the data series caused by public holidays which are included in the data series of Datastream or missing values, we consider solely the days when all three contracts have positive open interest and trading volume data. By estimating volatility after deleting the days when one or more of the futures contracts do not exhibit valid data, it is ensured that $\boldsymbol{v}_{\boldsymbol{t}-\mathbf{1 , t - 5}}$ and $\boldsymbol{v}_{\boldsymbol{t}-\mathbf{1 , t - 2 2}}$ in equation (1) refer always to identical historical time periods. Thus, we aim to eliminate any non- 
Table 1: Data characteristics

\begin{tabular}{lccccccccc}
\hline \multicolumn{1}{c}{ Descriptive statistics } & \multicolumn{3}{c}{ Correlation } \\
& Mean & SD & Skew & Kurt & Min & Max & $P K$ & $G K$ & $R S$ \\
\hline \multicolumn{10}{c}{ Natural Gas } \\
\hline$P K$ & 0.279 & 0.217 & 2.862 & 15.681 & 0.006 & 2.445 & 1.000 & & \\
$G K$ & 0.269 & 0.197 & 2.903 & 20.077 & 0.006 & 2.816 & 0.954 & 1.000 & \\
$R S$ & 0.262 & 0.202 & 3.783 & 39.623 & 0.007 & 3.486 & 0.851 & 0.966 & 1.000 \\
\hline \multicolumn{10}{c}{ Gasoil } \\
\hline$P K$ & 0.273 & 0.139 & 1.811 & 5.164 & 0.058 & 1.161 \\
$G K$ & 0.290 & 0.149 & 1.992 & 6.395 & 0.048 & 1.283 & 0.966 & 1.000 & \\
$R S$ & 0.294 & 0.161 & 2.266 & 9.246 & 0.040 & 1.700 & 0.914 & 0.982 & 1.000 \\
\hline \multicolumn{10}{c}{ Brent } \\
\hline$P K$ & 0.293 & 0.167 & 2.520 & 10.547 & 0.065 & 1.860 \\
$G K$ & 0.297 & 0.163 & 2.613 & 11.144 & 0.068 & 1.787 & 0.954 & 1.000 & \\
$R S$ & 0.292 & 0.167 & 2.650 & 11.193 & 0.048 & 1.701 & 0.876 & 0.977 & 1.000 \\
\hline
\end{tabular}

Note: $P K$ is the Parkinson estimator. $G K$ and $R S$ denote the Garman-Klass and Rogers-Satchell estimators, respectively. The sample period runs from May 2005 to June 2012.

synchronicity which may have a distorting effect on potentially existing volatility transmission patterns. In total, volatility data for 1812 trading days are available. Since the data emerge from the same market place, it is to be emphasized that the analysis does not need to cope with issues of overlapping trading and non-trading times, as for example in Lin and Tamvakis (2001).

Table 1 provides descriptive statistics for the daily annualized volatilities for all employed estimators. The volatility time series obtained with the three estimators have a very similar average magnitude per asset, especially for natural gas and Brent crude oil. The standard deviations reported in the third column show that the volatility of all three assets itself exhibits significant time series variation with natural gas (gasoil) being most (least) volatile. Furthermore, all estimates show positive skewness and large kurtosis and are thus in line with documented stylized facts about financial market volatility. Additionally, table 1 includes correlation matrices among the considered volatility proxies. It becomes obvious that they move closely together, as expected, with $P K$ and $R S$ showing lowest correlations (but still above 0.85 ) between each other.

Figure 1 illustrates the general volatility dynamics over the entire sample under 

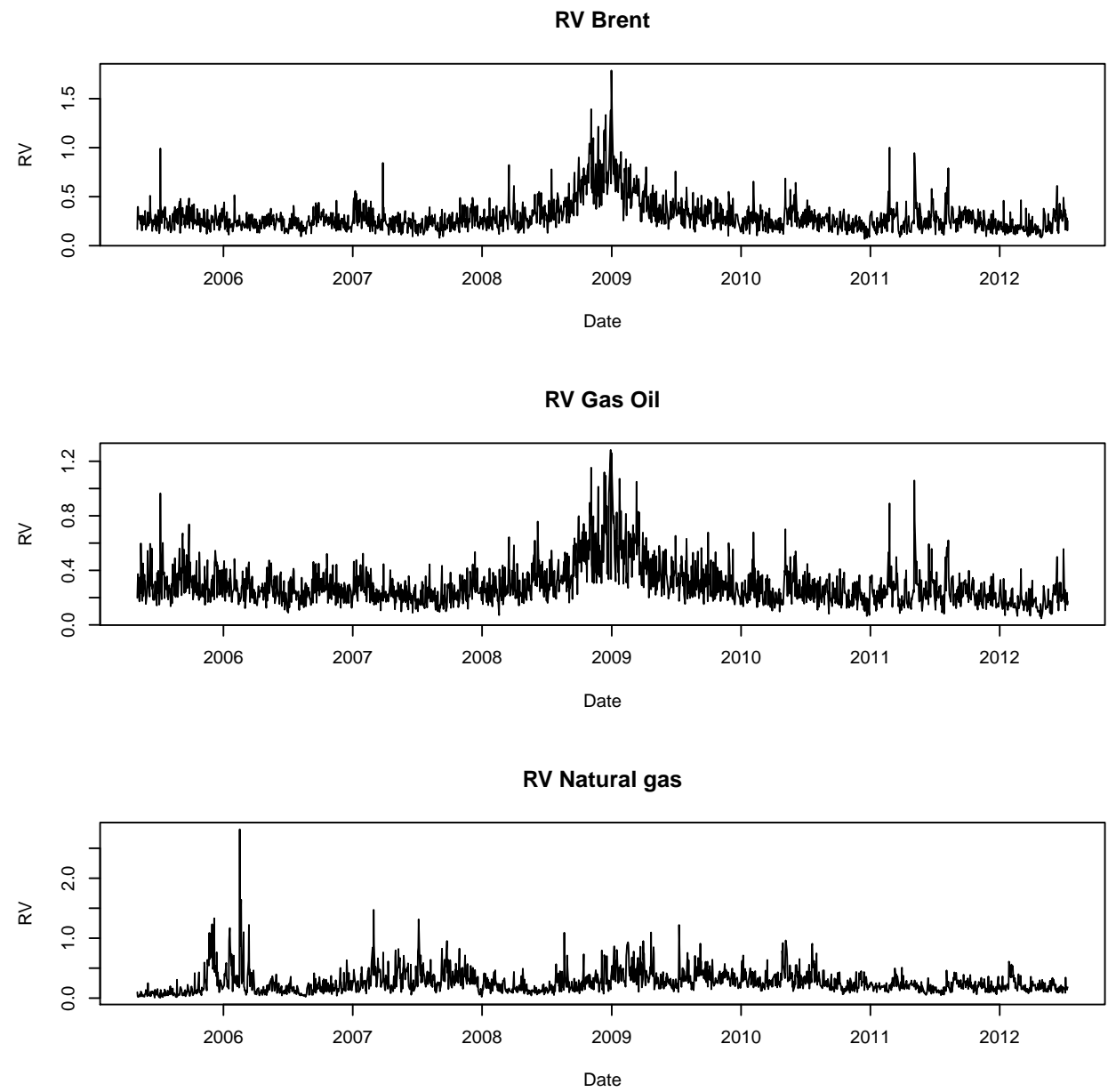

Figure 1: Plots of annualized daily volatility obtained with the Garman Klass $(G K)$ estimator

consideration for the case of $G K$. The volatility structures of Brent oil and gasoil seem to be similar, following major events experienced by global financial markets since 2005. In contrast, the volatility dynamics of natural gas shows a different pattern, probably more triggered by its seasonal demand and storage cycles. This observation is conform to the conclusion of Ewing et al. (2002) that natural gas return volatility responds more to events like supply interruptions and changes in reserves.

The stationarity of the univariate time series under consideration is a key assumption of the VHAR estimation and the tests for Granger causality. In the following, the logarithmic range-based volatilities are tested for stationarity with 
the Augmented Dickey-Fuller (ADF) test for unit roots, Phillips and Perron (1988) (PP) test and Elliott et al. (1996) (EPS) test. Results of all three unit root test show that the time series of the logarithmic volatility range-based estimates at hand are stationary and are summarized in table 2.

Table 2: Unit root tests

\begin{tabular}{lccc}
\hline & ADF & PP & ERS \\
& $H_{0}:$ unit root & $H_{0}$ : stationarity & $H_{0}:$ unit root \\
\hline Brent & -15.27 & Parkinson estimator \\
Gasoil & -16.16 & -26.50 & -15.19 \\
Natural gas & -14.51 & -27.53 & -14.64 \\
\hline \multicolumn{3}{c}{ Garman-Klass estimator } \\
\hline Brent & -14.65 & -25.34 & -13.77 \\
Gasoil & -13.71 & -27.04 & -12.28 \\
Natural gas & -16.23 & -20.48 & -12.24 \\
& & Rogers-Satchell estimator \\
\hline Brent & -15.89 & -27.40 & -10.30 \\
Gasoil & -17.27 & -28.72 & -15.28 \\
Natural gas & -14.48 & -22.17 & -12.26 \\
\hline
\end{tabular}

Note: Unit root tests are run for log range-based volatility. ADF is the Augmented Dickey-Fuller test. PP is the Phillips-Peron Test. ERS is the Elliott-Rothenberg-Stock test for stationarity. The t-values of the ADF and PP tests are compared with the Dickey-Fuller (DF) critical values. The critical values tabulated in Elliott et al. (1996) are used for to evaluate the EPS test. All test values are significant at the $1 \%$ level.

\section{Empirical results}

In this section, the results of the tests for Granger causality and the transmission models are presented, followed by a discussion of the dynamic correlation structure of the energy futures volatilities.

\subsection{Granger causality tests}

The Granger causality tests (Granger, 1969) are based on the full VHAR model presented in section 2. If $v_{k}$ does not Granger cause $v_{1}$, all of the coefficients on the volatility components $v_{t-1, k}, v_{t-1, t-5, k}$ and $v_{t-1, t-22, k}$ are zero in the equation for 
$v_{1}$. This linear restriction can be tested using Wald-statistics (Lütkepohl, 2005). Furthermore, because the relationship is not symmetric, Granger causality in one direction does not imply causality in the other direction.

Table 3: Multivariate Granger causality tests

\begin{tabular}{ccccccccccc}
\hline & & $P K$ & & \multicolumn{3}{c}{$G K$} & & \multicolumn{3}{c}{$R S$} \\
& Brent & Gasoil & Nat Gas & Brent & Gasoil & Nat Gas & Brent & Gasoil & Nat Gas \\
\hline Brent & - & 0.24 & 0.15 & - & 0.01 & 0.15 & - & 1.80 & 0.48 \\
& & $(0.63)$ & $(0.70)$ & & $(0.97)$ & $(0.70)$ & & $(0.18)$ & $(0.49)$ \\
Gasoil & 3.10 & - & 0.01 & 5.74 & - & 0.01 & 8.59 & - & 0.01 \\
& $(0.08)$ & & $(0.98)$ & $(0.02)$ & & $(0.93)$ & $(0.00)$ & & $(0.94)$ \\
\multirow{2}{*}{ Nat Gas } & 3.26 & 2.62 & - & 7.26 & 6.15 & - & 7.71 & 6.40 & - \\
& $(0.07)$ & $(0.11)$ & & $(0.01)$ & $(0.01)$ & & $(0.01)$ & $(0.01)$ & \\
\hline
\end{tabular}

Note: The first column represents endogenous variables in a single equation from the VHAR system. Columns 2 to 4 give the Granger causality F-values for pairs of equations within the system. The corresponding p-values of the F-statistics are shown in parentheses.

The data in table 3 summarizes the multi-equation F-tests for the equality of coefficients. The first column represents the endogenous variables (volatility components) in an equation from the VHAR system. The values are test statistics for the variables in the column to Granger-cause the variable in the raw. For instance, the F-value that the Brent volatility components based on different time periods Granger-causes today's gasoil Garman-Klass range-based volatility is 5.74, which is significant at the $2 \%$ level.

For all three range-based volatility estimates, very similar patterns in the Granger causality relationships can be observed. In the sample period under consideration, Brent crude oil volatility leads the volatility of gasoil and natural gas. This causality is significant at maximum of $2 \%$ significance level for $G K$ and $R G$ and at the $10 \%$ level for $P K$. This is a reasonable result, since crude oil is a large component of the gasoil production costs and there is a theoretical framework discussed above expecting linkage between natural gas an oil. Furthermore, gasoil volatility seems to have an impact on the future volatility of natural gas futures, with Granger causality being significant at $1 \%$ level for both $G K$ and $R S$ and around $10.5 \%$ for $P K$. Based on the results of the Granger causality tests, we now seek to gain further insights into the mechanism of volatility transmission by estimating a VHAR model 
comprising short-, medium- and long-term volatility components of all three energy futures under consideration.

\subsection{Regression results}

The estimation is started with the full model defined in the equation (1) and the best model is chosen stepwise using the Akaike information criterion (AIC). To save space, only the coefficients of the restricted models with the lowest AIC are reported. The regression results are summarized in table 4 and include estimations made with all three considered range-based volatility proxies for robustness purposes. $\beta_{i, B}(i=$ $1,5,22)$ denotes the previous daily, weekly and monthly logarithmic volatility for Brent crude oil, respectively. $\beta_{i, G}$ and $\beta_{i, N}$ label the corresponding variables for gasoil and natural gas futures contracts.

Starting with Brent, it appears that its volatility is affected mostly by the information in its own three components. The findings established with $R S$ suggest that additionally the short-term volatility component of gasoil might carry significant information for the current volatility of Brent. However, this cannot be confirmed using the other two volatility proxies. Across all three volatility estimators, the own long-term volatility component appears to be largest in terms of magnitude followed by the medium- and short-term components indicating that volatility over longer time intervals has a strong influence on volatility over shorter time intervals.

The current volatility level of gasoil, in turn, does not depend on its own short-term component, but in all three cases rather on the short-term volatility of Brent. This is an interesting result, confirming the findings arisen from the multivariate Granger causality tests. Furthermore, the volatility of gasoil futures depends significantly on its own medium- and long-term components. $R S$ suggests an additional medium-term impact by both other assets which is noticeably smaller in magnitude and significant only at the $10 \%$ level. In the case of gasoil, similarly to Brent, the own long-term volatility component exhibits the largest magnitude and highest t-values whereas the short-term component happens to be by far the least important out of all three in terms of magnitude. 
Table 4: Regression results

\begin{tabular}{|c|c|c|c|c|c|c|c|c|c|}
\hline & \multicolumn{3}{|c|}{$P K$} & \multicolumn{3}{|c|}{$G K$} & \multicolumn{3}{|c|}{$R S$} \\
\hline & Brent & Gasoil & Nat Gas & Brent & Gasoil & Nat Gas & Brent & Gasoil & Nat Gas \\
\hline$\beta_{0}$ & $\begin{array}{c}-0.15 \\
(-3.77)\end{array}$ & $\begin{array}{c}-0.17 \\
(-4.70)\end{array}$ & $\begin{array}{c}-0.19 \\
(-3.50)\end{array}$ & $\begin{array}{c}-0.16 \\
(-4.02)\end{array}$ & $\begin{array}{l}-0.17 \\
(-4.64)\end{array}$ & $\begin{array}{l}-0.20 \\
(-3.31)\end{array}$ & $\begin{array}{c}-0.16 \\
(-3.65)\end{array}$ & $\begin{array}{l}-0.18 \\
(-4.17)\end{array}$ & $\begin{array}{c}-0.21 \\
(-3.87)\end{array}$ \\
\hline$\beta_{1, B}$ & $\begin{array}{c}0.12 \\
(3.65)\end{array}$ & $\begin{array}{c}0.13 \\
(4.65)\end{array}$ & - & $\begin{array}{c}0.10 \\
(2.88)\end{array}$ & $\begin{array}{c}0.11 \\
(3.97)\end{array}$ & - & $\begin{array}{c}0.05 \\
(1.51)\end{array}$ & $\begin{array}{c}0.10 \\
(3.06)\end{array}$ & - \\
\hline$\beta_{5, B}$ & $\begin{array}{c}0.33 \\
(6.51)\end{array}$ & $\begin{array}{l}- \\
-\end{array}$ & - & $\begin{array}{c}0.34 \\
(6.65)\end{array}$ & $\begin{array}{l}- \\
-\end{array}$ & - & $\begin{array}{c}0.27 \\
(5.15)\end{array}$ & $\begin{array}{c}0.09 \\
(1.52)\end{array}$ & - \\
\hline$\beta_{22, B}$ & $\begin{array}{c}0.47 \\
(8.66)\end{array}$ & $\begin{array}{l}- \\
-\end{array}$ & $\begin{array}{c}0.31 \\
(2.31)\end{array}$ & $\begin{array}{c}0.47 \\
(8.81)\end{array}$ & $\begin{array}{l}- \\
-\end{array}$ & $\begin{array}{c}0.12 \\
(1.75)\end{array}$ & $\begin{array}{c}0.51 \\
(8.45)\end{array}$ & - & $\begin{array}{c}0.31 \\
(2.36)\end{array}$ \\
\hline$\beta_{1, G}$ & - & - & - & - & - & - & $\begin{array}{c}0.09 \\
(3.08)\end{array}$ & - & - \\
\hline$\beta_{5, G}$ & - & $\begin{array}{c}0.35 \\
(7.04)\end{array}$ & - & - & $\begin{array}{c}0.34 \\
(6.70)\end{array}$ & $\begin{array}{l}-0.10 \\
(-1.62)\end{array}$ & - & $\begin{array}{c}0.30 \\
(4.57)\end{array}$ & - \\
\hline$\beta_{22, G}$ & - & $\begin{array}{c}0.43 \\
(8.14)\end{array}$ & $\begin{array}{c}-0.29 \\
(-2.17)\end{array}$ & - & $\begin{array}{c}0.46 \\
(8.66)\end{array}$ & - & - & $\begin{array}{c}0.41 \\
(7.27)\end{array}$ & $\begin{array}{c}-0.29 \\
(-2.19)\end{array}$ \\
\hline$\beta_{1, N}$ & - & - & $\begin{array}{c}0.23 \\
(6.60)\end{array}$ & - & - & $\begin{array}{c}0.21 \\
(6.10)\end{array}$ & - & $\begin{array}{c}-0.03 \\
(-1.45)\end{array}$ & $\begin{array}{c}0.20 \\
(5.61)\end{array}$ \\
\hline$\beta_{5, N}$ & - & - & $\begin{array}{c}0.38 \\
(6.87)\end{array}$ & - & - & $\begin{array}{c}0.39 \\
(6.96)\end{array}$ & - & $\begin{array}{c}0.04 \\
(1.44)\end{array}$ & $\begin{array}{c}0.35 \\
(6.34)\end{array}$ \\
\hline$\beta_{22, N}$ & - & - & $\begin{array}{c}0.29 \\
(5.59)\end{array}$ & - & - & $\begin{array}{c}0.31 \\
(5.85)\end{array}$ & - & - & $\begin{array}{c}0.33 \\
(5.91)\end{array}$ \\
\hline $\begin{array}{l}d_{1} \\
d_{2}\end{array}$ & & $\begin{array}{l}0.0130 \\
0.9806\end{array}$ & & & $\begin{array}{l}0.0081 \\
0.9859\end{array}$ & & & $\begin{array}{l}0.0053 \\
0.9889\end{array}$ & \\
\hline
\end{tabular}

Note: Parameter estimates for the final restricted equations of the volatility transmission models are reported. $\beta_{i, B}(i=1,5,22)$ denotes the previous daily, weekly and monthly logarithmic volatility for Brent crude oil, respectively. $\beta_{i, G}$ and $\beta_{i, N}$ label the corresponding variables for gasoil and natural gas futures contracts. The best model is established stepwise using the AIC. The corresponding Newey-West t-statistics are given in parentheses. $d_{1}$ and $d_{2}$ are the parameters in (5).

The volatility equation for natural gas reveals a slightly different picture. In relation to the short- and medium-term volatility impact, only the own components seem to carry relevant information for its current volatility level. In the long-term contest, a significant positive impact is attributed, besides to $\beta_{22, N}$, also to Brent. Furthermore, $P K$ and $R S$ identify a similarly large but negative impact of the longterm variance component of the gasoil. In contrast to the other two assets, the largest importance in terms of magnitude is attributed to the own medium-term component. It is closely followed by the long-term components $\beta_{22, B}$ and $\beta_{22, N}$ whose magnitudes move closely together (except for the case of $G K$ ) but the own component $\beta_{22, N}$ shows consistently higher t-values. Again, the own short-term volatility component is significant but exhibits the lowest magnitude.

To sum up, the regression results document the existence of volatility spillovers in the ICE energy market and reveal interesting insights in the structure of the relationship. The source of the causality between Brent oil and gasoil is the short-term 
volatility of crude oil. While the middle and long-term components in the gasoil volatility are incorporated in its own time series, short-term shocks are determined solely by the fluctuation in the crude oil prices. Because crude oil is the major production component of gasoil, the volatility spillover can be interpreted as a reaction of the investors to sudden shocks in the production cost.

The findings for natural gas and crude oil suggest that the major source of the volatility transmission is the long-term volatility component; i.e., acting of longterm agents and the expected future size of trends and risk, as indicated by Corsi (2009), in the commodity time series. The short-term volatility includes significant information for its own future daily volatility development, but has no or only a weak impact on the volatility of the other considered energy futures. Brent oil seems broadly unaffected by volatility of gasoil and natural gas, but for all three estimators the long-term volatility of crude oil has an effect on the daily volatility of natural gas. Because both products are potential substitutes as an energy source or could be even linked through some pricing specifications as mentioned above, it is reasonable to expect that the origin of the relationship might be in the long-term expected future trends and risk. Furthermore, the long-term $(P K, R S)$ and the medium-term $(G K)$ volatility has a negative contribution to the future natural gas volatility. The fact that Brent crude oil volatility is leading other energy futures on the ICE seems plausible as well, since the oil futures exhibit significantly larger trading volumes and open interest. Overall, the regression results substantiate the interpretation of the Granger causality test results.

A further interesting aspect regards the comparison of our results with the findings of Bubák et al. (2011) who first use a similar multivariate time-series specification to document and examine volatility spillovers among Central European currencies and the EUR/USD foreign exchange. The authors establish that each currency has a different volatility transmission pattern but in terms of magnitude, the largest impact is attributed in most cases to the own medium-term volatility component. A crucial point for explaining the shift of the most important volatility component towards the long-term element may be the nature of the explored 
markets. Presumably, energy futures market data consistently exhibit a more pronounced dependence on its own monthly volatility due to storage cost and in this sequel, convenience yield effects which are not represented in the spot forex markets but are generally of persistent nature and hence not negligible when studying commodity markets.

\subsection{Conditional correlations}

In the text which follows, the evolution of pairwise dynamic conditional correlations between the range-based volatility estimates is discussed. The estimations are based on the DCC-GARCH model by Engle (2002) introduced in section 2.1. The DCC curve obtained with the Garman-Klass estimator is plotted in figure 2. The results gained by using the remaining estimators allow for a qualitatively very similar interpretation and the figures were omitted in order to save space. For all three volatility proxies the condition holds that $d_{1}+d_{2}<1$.

For all pairs, a time-varying and volatile pattern is present in the correlation structure. However, the conditional correlations for Brent oil vs. natural gas, and gasoil vs. natural gas remain bounded only between -0.15 and 0.15 , with a small peak observable in the period between 2008 and 2009, which is a reasonable result in the backdrop of the recent turmoil in the global financial markets. The Brent oil vs. gasoil correlation, on the other hand, has a clear positive magnitude bounded between 0.65 and 0.85 . Recently, the DCC curve fluctuates around 0.8, which is an obvious sign for the strong instantaneous relationship between the volatility time series. The evolution of the curve shows a continuously rising magnitude of the relationship between the volatilities of the two energy futures. While the correlation fluctuates between 0.65 and 0.75 before 2008, after 2008 the correlation remains mostly over 0.8. The DCC curve does not exhibit any significant spikes; solely a small peak is observable at the beginning of 2008, which occurs simultaneously with similar peaks in the remaining curves. This is most presumably related to the rising uncertainty in the financial and commodity markets related to the Lehman crash and the following financial distress. Overall, the pairwise DCC analysis reveals 
interesting aspects of volatility interdependencies, conform to the reported regression results.
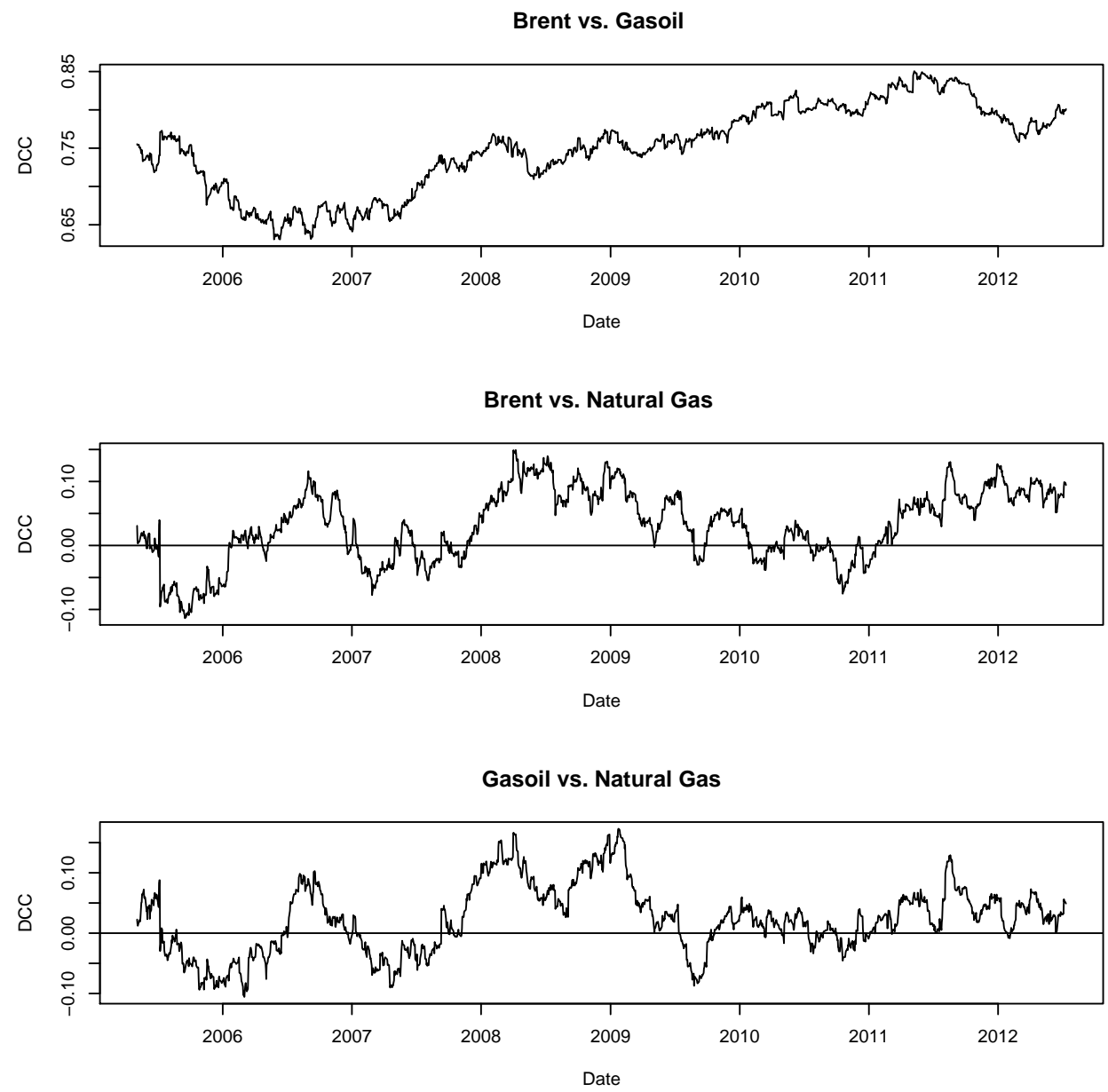

Figure 2: Plots of conditional correlations implied by the DCC model using the Garman-Klass $(G K)$ volatility estimator

\section{Conclusion}

This study is novel in the application of a multivariate HAR model with rangebased volatility estimates for studying volatility transmission patterns in energy futures market data. Splitting volatility in three components defined over different time horizons allows for gaining additional insights in the nature of volatility comovements, compared to commonly used GARCH related approaches. This paper 
is the first to utilize this methodology for the energy markets dataset. In particular, volatility transmission patterns are studied for nearest maturity futures on crude oil, gasoil and natural gas traded at the ICE. Crude oil is an essential component of production costs for gasoil which supposes the existence of significant co-movements in prices and volatilities of related financial products. Natural gas and crude oil, on the other hand, are to some extent substitutes as an energy source and are possibly linked via pricing specific of the ICE; hence it is reasonable to assume interdependences between their price fluctuations. As proxies for daily realized volatility, the range-based estimators of Parkinson (1980), Garman and Klass (1980) and Rogers and Satchell (1991) are employed. All three allow for qualitatively very similar interpretation of the results and confirm the robustness of the findings. Considering range-based estimators within the HAR framework is relatively seldom in the literature on financial market volatility.

The regression results support the interpretation of the Granger causality tests, largely documenting the existence of volatility spillovers in the energy futures market. In the case of Brent oil and gasoil ICE futures, the own historical long-term components appear to have the largest impact on current volatility in terms of magnitude whereas the strongest impact on natural gas volatility is ascribed to its own mid-term component. Moreover, crude oil volatility seems to lead the volatility of gasoil and natural gas. The causality between crude oil and gasoil appears to arise from the short-term volatility of Brent oil since short-term shocks are determined solely by the fluctuation in the crude oil futures prices. This volatility transmission effect may be interpreted as a investors' reaction to shocks in production costs. On the other hand, for natural gas and crude oil, the major source of volatility transmission appears to be the monthly volatility component caused by long-term agents. Brent oil seems broadly unaffected by the variation of its potential substitutes, whereas the long-term volatility of crude oil has an effect on the daily volatility of natural gas. Hence, the daily ranges of Brent crude oil futures carry significant information for the volatility evolution of other energy futures on the ICE. The pairwise dynamic conditional correlations analysis supports our conclusions. There is a 
strong positive instantaneous correlation between the oil and gasoil futures volatility, while the remaining two pairwise correlations fluctuate around zero. Overall, the results are relevant for research on investment strategies and thus of a high practical importance.

\section{References}

Ait-Sahalia, Y., Mancini, L., 2008. Out of sample forecasts of quadratic variation. Journal of Econometrics 147, 17-33.

Alizadeh, S., Brandt, M.W., Diebold, F.X., 2002. Range-based estimation of stochastic volatility models. Journal of Finance 57, 1047-1091.

Andersen, T.G., Bollerslev, T., 1998. Answering the skeptics: Yes, standard volatility models do provide accurate forecasts. International Economic Review 39, 885905 .

Andersen, T.G., Bollerslev, T., Diebold, F.X., 2007. Roughing it up: Including jump components in the measurement, modeling and forecasting of return volatility. Review of Economics and Statistics 89, 701-720.

Blair, B.J., Poon, S., Taylor, S.J., 2001. Forecasting S\&P 100 volatility: The incremental information content of implied volatilities and high-frequency index returns. Journal of Econometrics 105, 5-27.

Bollerslev, T., 1986. Generalised autoregressive conditional heteroskedasticity. Journal of Econometrics 32, 307-327.

Bubák, V., Kočenda, E., Žikeš, F., 2011. Volatility transmission in emerging European foreign exchange markets. Journal of Banking \& Finance 35, 2829-2841.

Chang, C.-L., McAleer, M., Tansuchat, R., 2010. Analyzing and forecasting volatility spillovers, asymmetries and hedging in major oil markets. Energy Economics $32,1445-1455$. 
Chevallier, J., 2012. Time-varying correlations in oil, gas and CO2 prices: An application using BEKK, CCC and DCC-MGARCH models. Applied Financial Economics 44, 4257-74.

Corsi, F., 2009. A simple approximate long-memory model of realized volatility. Journal of Financial Econometrics 7, 174-196.

Corsi, F., Mittnik, S., Pigorsch, C., Pigorsch, U., 2008. The volatility of realized volatility. Econometric Reviews 27, 46-78.

Elliot, G., Rothenberg, T.J., Stock, J.H., 1996. Efficient tests for an autoregressive unit root. Econometrica 64, 813-836.

Engle, R., 2002. Dynamic conditional correlation: A simple class of multivariate generalized autoregressive conditional heteroskedasticity models. Journal of Business and Economic Statistics 20, 339-350.

Ewing, B.T., Malik, F., Ozfidan, O., 2002. Volatility transmission in the oil and natural gas markets. Energy Economics 24, 525-538.

Ewing, B.T., Malik, F., 2013. Volatility transmission between gold and oil futures under structural breaks. International Review of Economics and Finance 25, 113121.

Garman, M.B., Klass, M.J., 1980. On the estimation of security price volatilities from historical data. Journal of Business 53, 67-78.

Ghoshray, A., 2011. Trends, persistence, and volatility in energy markets. ADB Economics Working Paper Series No. 275.

Granger, C.W.J., 1969. Investigating causal relations by econometric models and cross-spectral methods. Econometrica 37, 424-438.

Jacob, J., Vipul, 2008. Estimation and forecasting of stock volatility with rangebased estimators. The Journal of Futures Markets 28, 561-581. 
Jin X., Lin, S.X., Tamvakis, M. 2012. Volatility transmission and volatility impulse response functions in crude oil markets. Energy Economics, in press.

Khalifa, A.A.A., Hammoudeh, S., Otranto, E., Ramchander, S., 2012. Volatility transmission across currency, commodity and equity markets under multi chain regime switching: Implications for Hedging and Portfolio Allocation. CRENoS Working Paper 2012/14.

Lanza, A., Manera, M., McAleer, M., 2006. Modeling dynamic conditional correlations in WTI oil forward and futures returns. Finance Research Letters 3, 114-132.

Lin, S.X., Tamvakis, M.N., 2001. Spillover effects in energy futures markets. Energy Economics 23, 43-56.

Lütkepohl, H., 2005. New introduction to multiple time series analysis. Springer, Berlin.

Malik, F., Hammoudeh, S., 2007. Shock and volatility transmission in the oil, US and Gulf equity markets. International Review of Economics and Finance 16, $357-368$.

McAleer, M., Medeiros, M., 2008. Realized volatilty: A review. Econometric Reviews $26,10-45$.

Mu, X., 2007. Weather, Storage, and Natural Gas Price Dynamics: Fundamentals and Volatility. Energy Economics 29, 46-63.

Müller, U., Dacorogna, M., Dav, R., Olsen, R., Pictet, O., Weizsacker, J.v., 1997. Intraday trading volume and return volatility of the DJIA stocks: A note. Journal of Banking and Finance 27, 2035-2043.

Parkinson, M., 1980. The extreme value method for estimating the variance of the rate of return. Journal of Business 53, 61-65.

Pindyck, R.S., 2004. Volatility and commodity price dynamics. The Journal of Futures Markets 24, 1029-1047. 
Phillips, P.C.B., Perron, P., 1988. Testing for a unit root in time series regression. Biometrika 75, 335-346.

Rogers, L., Satchell, S., 1991. Estimating variance from high, low and closing prices. Annals of Applied Probability 1, 504-512.

Serra, T., 2011. Volatility spillovers between food and energy markets: A semiparametric approach. Energy Economics 33, 1155-1164.

Shu, J., Zhang, J.E., 2006. Testing range estimators of historical volatility. The Journal of Futures Markets 26, 297-313.

Silvennoinen, A., Teräsvirta, T., 2009. Multivariate GARCH models. In: Andersen, T., Davis, R., Kreiß, J.-P. Mikosch, T. (Eds.), Handbook of Financial Time Series. Springer, New York pp. 201-229.

Singh, A., Karali, B., Ramirez, O.A., 2011. High price volatility and spillover effects in energy markets. 2011 Annual Meeting, Pittsburgh, Pennsylvania, Agricultural and Applied Economics Association.

Soriano, P., Climent, F.J., 2006. Volatility transmission models: A survey. Revista de Economia Financiera 10, 32-81.

Vacha, L., Barunik, J., 2012. Co-movement of energy commodities revisited: Evidence from wavelet coherence analysis. Energy Economics 34, 241-247.

Vipul, Jacob, J., 2007. Forecasting performance of extreme-value volatility estimators. The Journal of Futures Markets 27, 1085-1105.

Yang, D., Zhang, Q., 2000. Drift-independent volatility estimation based on high, low, open, and close prices. Journal of Business 73, 477-491. 